\title{
SEEDING OF NEOCLASSICAL TEARING MODES BY INTERNAL CRASH EVENTS IN THE ASDEX UPGRADE AND DIII-D TOKAMAKS
}

\author{
V. Igochine ${ }^{1}$, A. Gude ${ }^{1}$, S. Günter ${ }^{1}$, J. M. Hanson ${ }^{2}$, K. Lackner ${ }^{1}$, C. Paz-Soldan ${ }^{3}$, E. Strait ${ }^{3}$ \\ H. Zohm ${ }^{1}$, The ASDEX Upgrade Team and The DIII-D Team \\ ${ }^{1}$ Max-Planck Institute for Plasma Physics \\ Garching bei München, Federal Republic of Germany \\ ${ }^{2}$ Department of Applied Mathematics and Applied Physics, Columbia University \\ New York, United States of America \\ ${ }^{3}$ General Atomics, P.O. Box 85608 \\ San Diego, California, United States of America
}

Email: valentin.igochine@ipp.mpg.de

Keywords: seeding, tearing, reconnection

\begin{abstract}
Seeding of neoclassical tearing modes (NTMs) by perturbations from other MHD instabilities (sawteeth, fishbones, ELMs, etc) is one of the main MHD problems which has to be avoided or controlled in ITER. NTMs can lead to strong degradation of plasma confinement or even to a disruption. This paper compares the seeding of $(3,2)$ neoclassical tearing modes in DIII-D and ASDEX Upgrade tokamaks. It was found in both devices that a mode can start as an ideal kink mode that converts into a tearing mode on a time-scale much longer $\left(\sim 10^{-2}\right)$ than the duration of the trigger event $\left(\sim 10^{-4}\right)$. These findings are in good agreement with those for $(2,1)$ mode seeding in ASDEX Upgrade as well as with non-linear MHD simulations. This result revises the simplified picture of fast island formation occurring only during the trigger event.
\end{abstract}

\section{INTRODUCTION}

NTM formation triggered by perturbations from other MHD instabilities like sawteeth, fishbones, or ELMs, is one of the most important MHD processes that result in a big island structure and confinement degradation in tokamaks. This type of tearing mode seeding is considered the most important for future fusion reactors like ITER [1] because internal events provide strong magnetic perturbations able to trigger the mode at small normalized pressure values $\left(\beta_{N}=\beta\left(a B_{t} / I_{p}\right), \beta=2 \mu_{0}\langle p\rangle / B_{t}^{2} ;\langle p\rangle\right.$ is the volume average pressure, $B_{t}$ is the vacuum toroidal magnetic field at the axis, $a$ is the minor radius and $I_{p}$ is the plasma current). Several papers from different tokamaks investigate conditions for NTM onset based on analysis of global and local plasma parameters. These studies lead to empirical scaling for NTM onset conditions [2-5]. Other papers were focused on frequency analysis of NTMs and seed perturbation $[5,6]$ or on the influence of a particular quantity on the mode onset, for example the influence of the plasma rotation on the NTM onset [7]. In the present work, the main 
attention is paid to detailed analysis of the seeding process based on local high time resolution measurements around the resonant surface. The considered discharges represent standard experimental conditions. The main questions which we try to clarify are the exact details of the seeding process and the importance of different factors for this. The process of tearing mode formation during the seeding process implies magnetic reconnection at the rational surface, which is studied mainly based on electron cyclotron emission measurements (ECE). Such reconnection has been investigated in great detail in the ASDEX Upgrade tokamak for the seeding of $(\mathrm{m}, \mathrm{n})=(2,1)$ modes ( $\mathrm{m}$ and $\mathrm{n}$ are poloidal and toroidal mode numbers respectively) $[8,9]$. It was shown that an internal crash event can lead to a dominantly ideal kink mode at the resonant surface. This mode transforms into a tearing mode on a much longer timescale $\left(10^{-3} s-10^{-2} s\right)$ than the crash itself $\left(10^{-5} s-10^{-4} s\right)$. The reason is the two different timescales for plasma deformations: (i) an ideal kink deformation is possible at fast timescales (Alfven time, $\tau_{A} \sim 1 \mu \mathrm{s}$ ); (ii) an island formation requires changes of the field line topology and longer time (resistive timescales, Sweet-Parker single fluid, resistive MHD time: $\tau_{S P} \sim 0.1 s$ ). Real fusion plasmas are collisionless and the effective resistive time is much smaller compared to the Sweet-Parker prediction, but it is still larger than the Alfven time (see details in Appendix of Ref. [9]).

The main aim of this work is to compare the seeding process in two different tokamaks for the same tearing mode and check the existence of a dominantly ideal mode after the crash. In our previous investigations in ASDEX Upgrade, we have looked into cases of $(2,1)$ mode seeding using Electron Cyclotron Emission (ECE) measurements with high radial resolution and low noise. This mode is also expected to be one of the main problems for ITER. Unfortunately, for the typical discharges with $(2,1)$ modes in DIII-D ECE measurements do not cover the region of interest sufficiently due to density cutoff of the diagnostic. Thus, a direct comparison of $(2,1)$ mode seeding in two tokamaks is not possible. We, therefore, compare the seeding of $(3,2)$ modes, which is often well observed by ECE diagnostics in both ASDEX Upgrade and DIII-D. The data quality for $(3,2)$ mode seeding is not so good as in the dedicated experiments with $(2,1)$ modes where the mode is typically larger and the ECE is adjusted for optimal measurements at the mode location (lower noise and better coverage). Thus, new algorithms for the data analysis are required. These methods are described in section 2 . Importance of the plasma rotation profiles and ideal MHD limits for the seeding are discussed in section 3 . 
2. IDEAL AND RESISTIVE CHARACTER OF SEEDED $(3,2)$ MODES IN DIII-D AND ASDEX UPGRADE TOKAMAKS

As was mentioned before, the $(3,2)$ mode is chosen because it is covered by ECE in many discharges in both tokamaks. Compared to dedicated $(2,1)$ mode discharges in ASDEX Upgrade [8,9], there is a larger space separation between ECE channels and higher noise. As a result, the previous method of island identification directly from ECE measurements (see figure 1 in [8]) is not applicable and a new type of data analysis is required. The quality of raw ECE measurements is not sufficient to deduce a phase jump at the resonant surface, which is the standard signature of a tearing mode (Chapter 3 in Ref. [10]), directly from the signals. Instead, a Fourier based method with a magnetic signal as a reference is applied. The program traces the maximum amplitude in the spectrogram of the magnetic signal and determines phases and amplitudes of the ECE signals at this specific frequency. A similar method has already been used in algorithms for online NTM identification and feedback stabilization of NTMs [11, 12]. We use sliding Fast Fourier Transformation (FFT) with a relatively large time window $\left(T_{F F T}=\right.$ $1.5 \mathrm{~ms}$ ), for better noise reduction. The time shift of the FFT window is eight times smaller $\left(\Delta t_{F F T}=0.19 m s\right)$. Variation of these parameters up to factors 2-3 does not influence our results. Furthermore, relative changes of the mode amplitude are determined from the magnetic signal: $A_{(3,2)} \sim \sqrt{b_{(3,2)}}$, where $b_{(3,2)}$ is the measured perturbation amplitude at the $(3,2)$ mode frequency from magnetic signal. 
a)
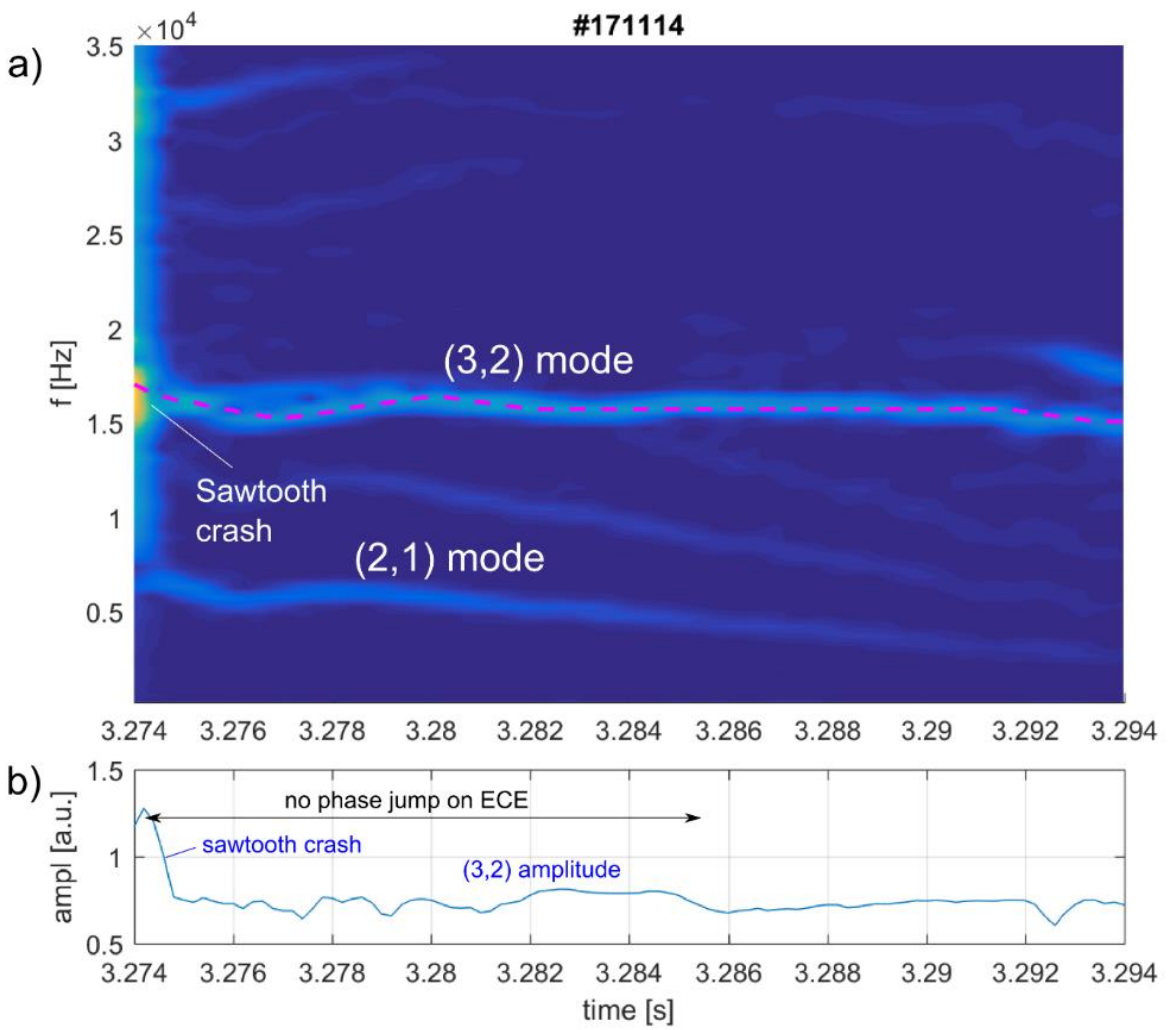

Figure 1. (a) Spectrogram of the magnetic signal during the seeding event in the DIII-D tokamak for discharge \#171114. The sawtooth crash produces strong $(3,2)$ and weak $(2,1)$ mode. The dashed red line shows the frequency tracking of the strongest mode, which is the $(3,2)$ mode after the crash, by our algorithm. (b) The amplitude of the tracked mode. The time window without phase jump on ECE is indicated.

A spectrogram of the magnetic signal for a $(3,2)$ mode triggered by a sawtooth crash on DIII$\mathrm{D}$ is shown in figure 1a. The algorithm traces the mode frequency robustly (dashed red line). The amplitude of the $(3,2)$ mode, determined from the magnetic signal, is large directly after the crash and remains nearly constant during the whole time window (figure 1b). The filtered phase and amplitude of the ECE signals are shown in figures 2a-2c. Position of the ECE channels are marked by circles at the left side of the figures. The phase jump at the resonant surface is identified by two different methods. The first method determines the relative phases of all signals with respect to a reference signal as shown in figure $2 \mathrm{a}$. The reference is chosen at or slightly inside the $(3,2)$ resonance surface and it has zero phase by definition (this channel has pure blue color in figure 2a). The figures show a cell-type representation of phase and amplitude information from ECE at the mode frequency. The vertical cell size corresponds to 
the distance between neighboring ECE channels in $\rho_{p o l}$. The horizontal cell size has the size of the FFT time shift $\left(\Delta t_{F F T}\right)$, which is several times smaller compared to the size of the FFT window (shown in red in figure $2 \mathrm{~d}$ ). The cell color represents the phase or the amplitude for the FFT time window centered at the cell center. The first indication of different phases around the resonant surface (the dashed red line in figure 2) becomes visible around $3.287 \mathrm{~s}$, which is well after the triggering event at 3.274s. Thus, in spite of the relatively large amplitude detected by the magnetic measurement, there is a long phase (about $10^{4} \tau_{A}$ ) of a dominantly ideal mode without a phase jump at the resonant surface (figure 1b). At the same time, this delay is one order of magnitude faster than the simple Sweet-Parker prediction mentioned above. The second method for the phase determination considers the phase differences between neighboring ECE channels. In this case, color represents the phase between two neighboring ECE channels (figure $2 b$, showing the occurrence of the phase jump at approximately the same time around 3.287s). Amplitudes of the ECE signals are shown in figure 2c, the reference magnetic signal in figure $2 \mathrm{~d}$.

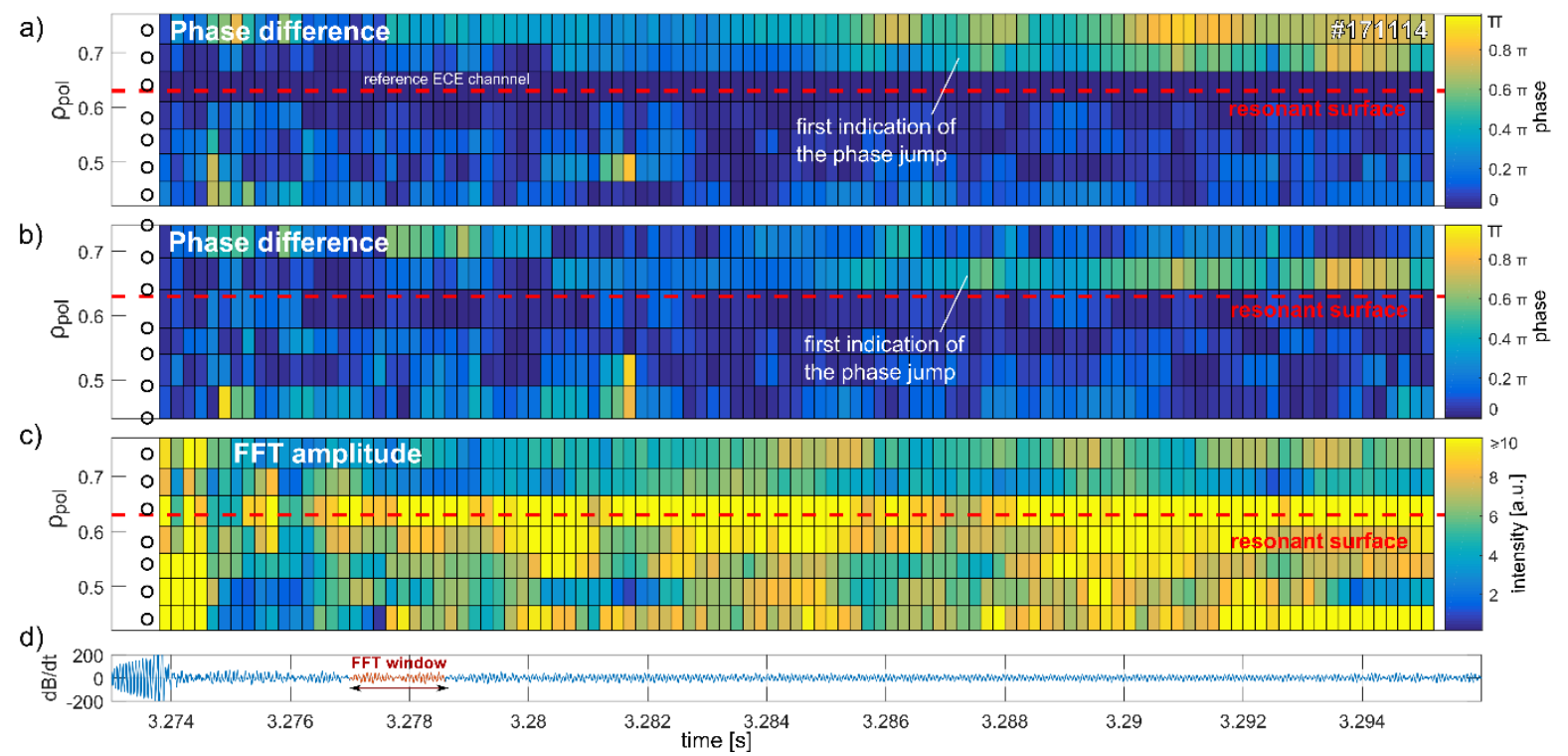

Figure 2. Identification of the ECE phase jump at the resonant surface by sliding FFT algorithm (DIII-D, \#171114). Circles at the left sides of figures $2 \mathrm{a}-2 \mathrm{c}$ correspond to the position of ECE channels. The $(3,2)$ resonant surface is marked by the dashed red line. Color bars show values of the phase or amplitude. (a) The phase difference between the reference channel around $\rho_{p o l}=0.62$ and the other channels at the mode frequency; (b) The phase difference between neighboring channels at the mode frequency; (c) The FFT Amplitude at the mode frequency; (d) magnetic signal with indicated time window size used for FFT transformation. 


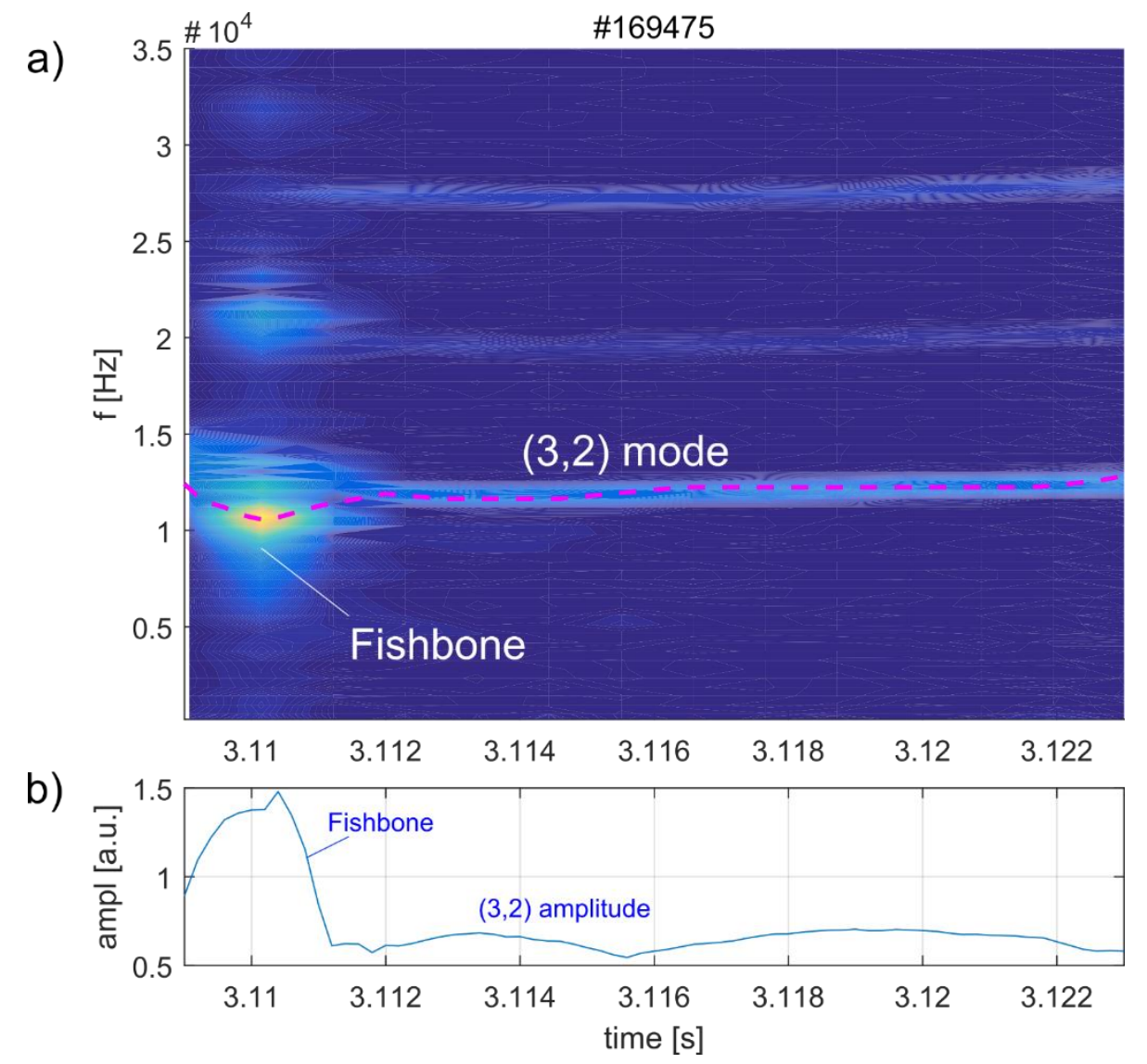

Figure 3. (a) Spectrogram of the magnetic signal during the seeding event in DIII-D tokamak for discharge \#169475. The fishbone produces strong $(3,2)$ mode directly after the fishbone. The dashed magenta line shows the tracking of the strongest mode, which is the $(3,2)$ mode after the fishbone. (b) The amplitude of the tracked mode.

An example for the second typical class from DIII-D is shown in figure 3. The mode is triggered here by a $n=1$ fishbone. The $(3,2)$ mode amplitude is also large in this case directly after the fishbone (figure $3 b$ ). At the same time, the phase jump is visible immediately after the seeding event in both variants of ECE phase analysis as shown in figures $4 \mathrm{a}$ and $4 \mathrm{~b}$. Thus, the island formation is much faster in this case and no transition can be identified with our analysis. If the island formation is delayed, this delay must be shorter than the FFT window $\left(T_{F F T}=1.5 \mathrm{~ms}\right)$. The size of the FFT window is given in figure $4 \mathrm{~d}$. 


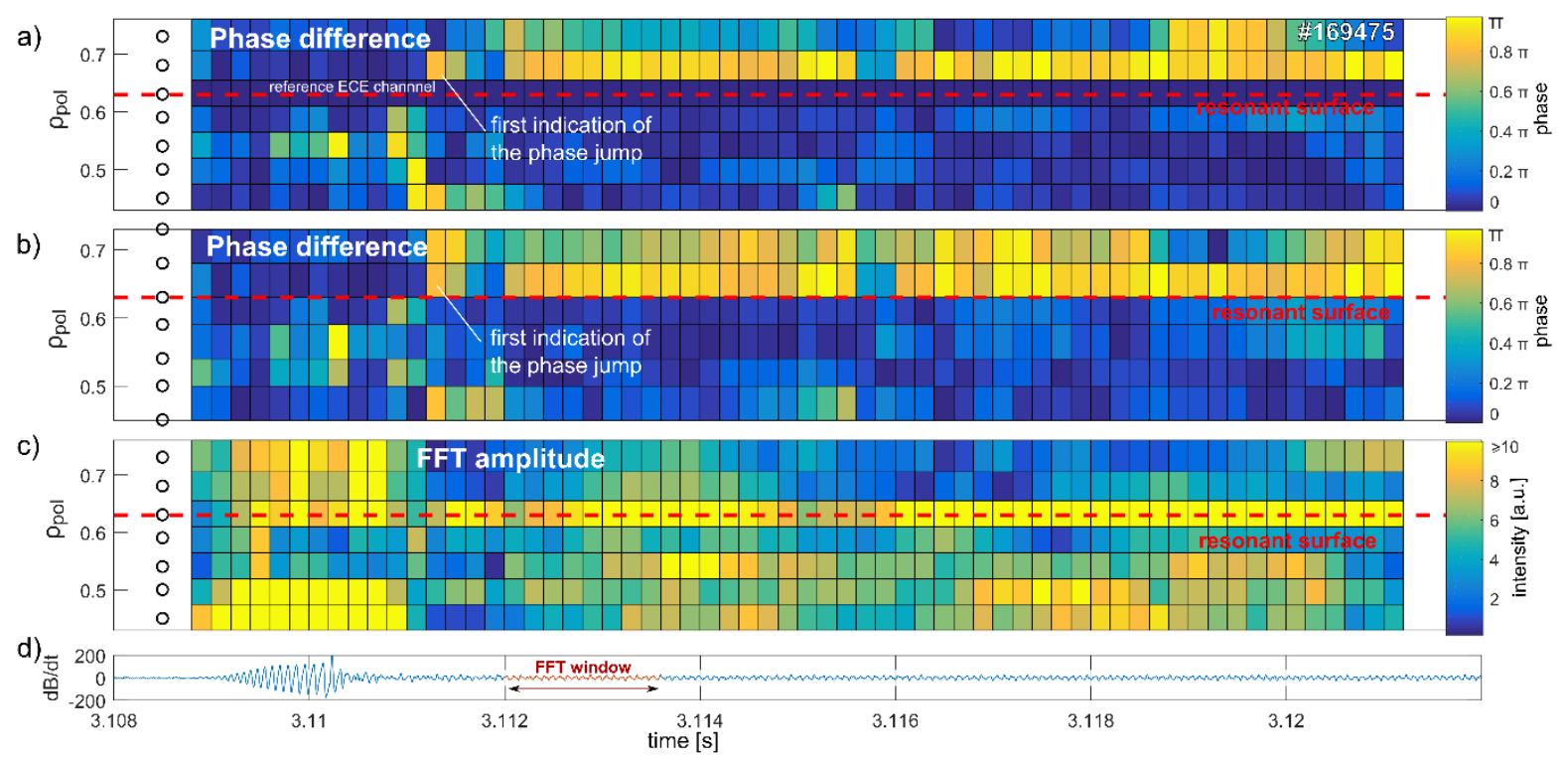

Figure 4. Identification of the ECE phase jump at the resonant surface by sliding FFT algorithm (DIII-D, \#169475). Circles at the left sides of figures $4 a-4 c$ correspond to the position of ECE channels. The resonant surface for $(3,2)$ mode is marked by the dashed red line. Color bars show values of the phase or amplitude. (a) The phase difference between the reference channel around $\rho_{\text {pol }}=0.62$ and the other channels at the mode frequency; (b) The phase difference between neighboring channels at the mode frequency; (c) The FFT Amplitude at the mode frequency; (d) magnetic signal with indicated time window size used for FFT transformation. 


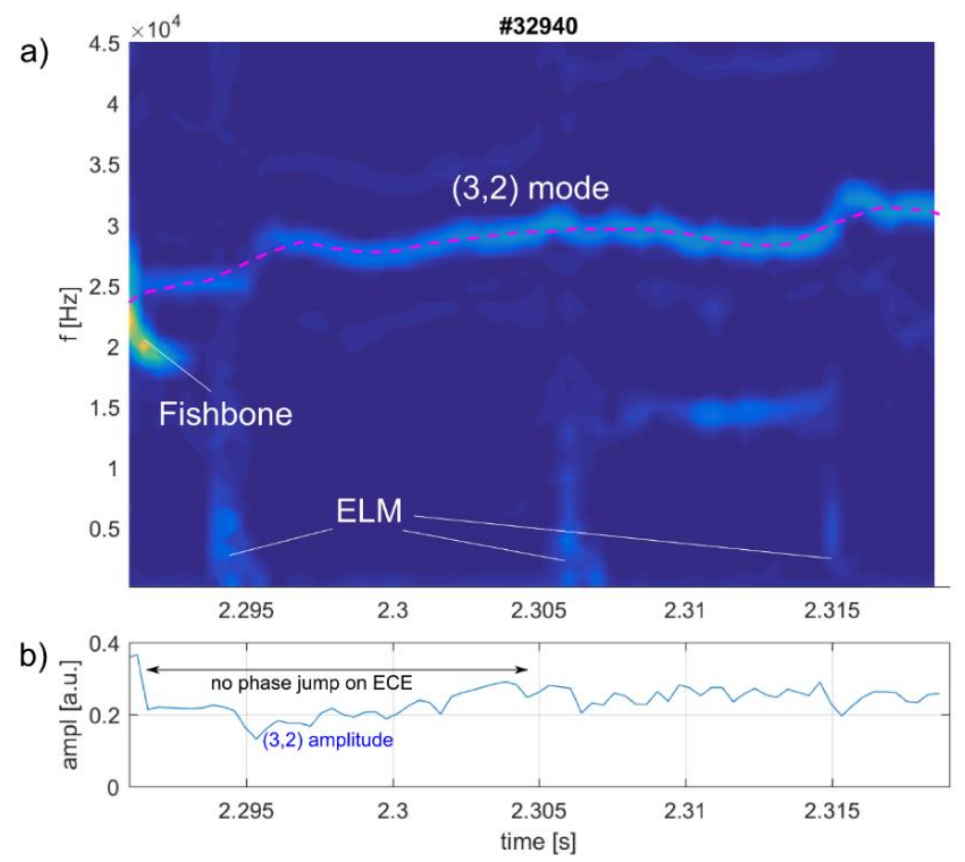

Figure 5. (a) Spectrogram of the magnetic signal during the seeding event in ASDEX Upgrade tokamak for discharge \#32940. The fishbone produces a strong $(3,2)$ mode directly after the seeding event. The dashed magenta line shows the tracking of the strongest mode, which is the $(3,2)$ mode after the fishbone. (b) Amplitude of the tracked mode. The time window without phase jump on ECE is indicated.

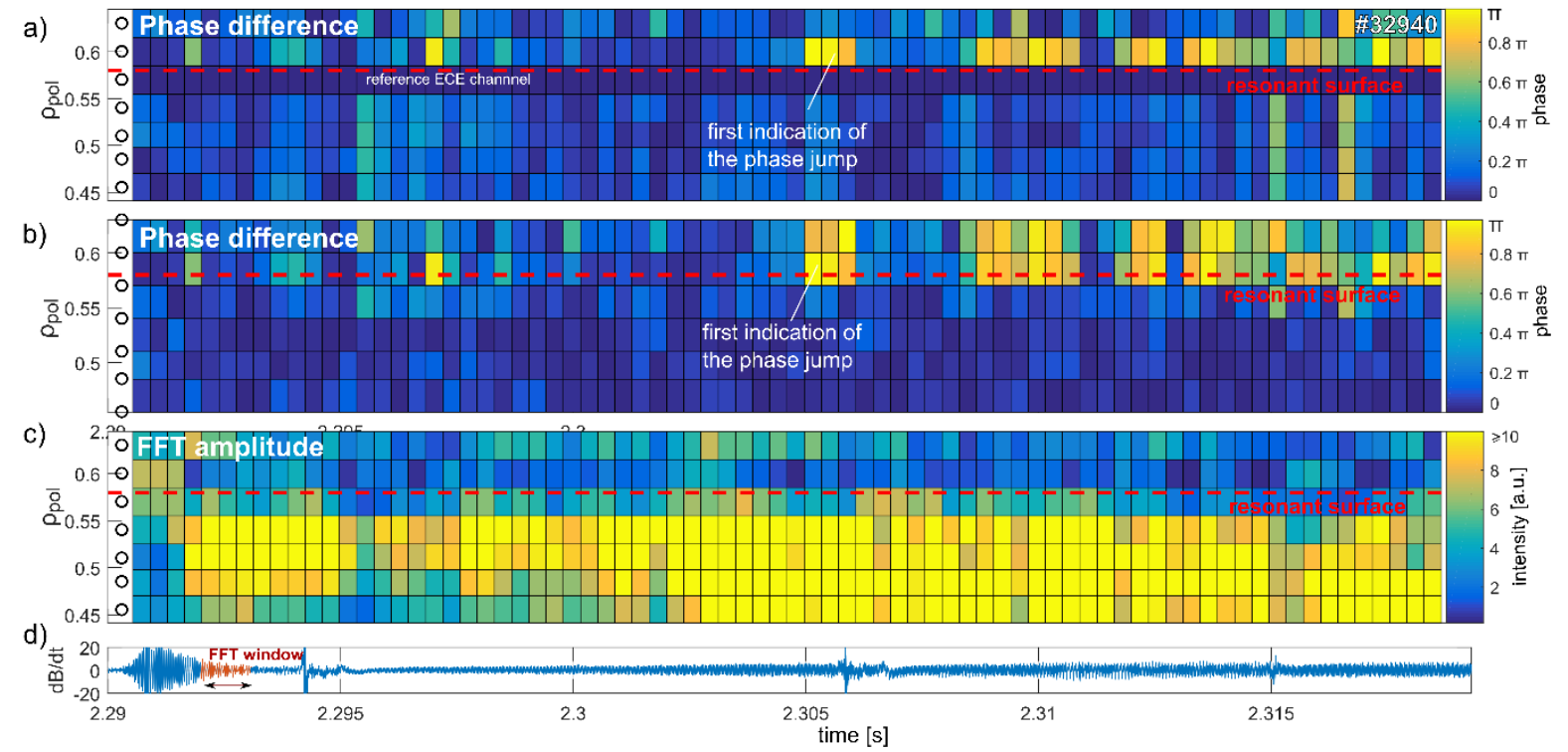

Figure 6. Identification of the ECE phase jump at the resonant surface by sliding FFT algorithm (AUG, \#32940). Circles at the left sides of figures 6a-6c correspond to the position of ECE channels. The resonant surface for $(3,2)$ mode is marked by the dashed red line. Color bars show values of the phase or amplitude. (a) The phase difference between the reference channel around $\rho_{p o l}=0.56$ and the other channels at the 
mode frequency; (b) The phase difference between neighboring channels at the mode frequency; (c) The FFT Amplitude at the mode frequency; (d) magnetic signal with indicated time window size used for FFT transformation.

Application of this analysis to a typical discharge from ASDEX Upgrade is shown in figures 5 and 6. A clear time window without phase jump is observed directly after the $(3,2)$ seeding by a $n=1$ fishbone. The mode amplitude measured directly after the crash by the magnetic coils is large (figure $5 b$ ). Thus, the dominant part of the mode has ideal character directly after the seeding. These observations are similar to the DIII-D case with slow conversion shown in figures 1 and $2\left(\tau_{c o n v} \approx 10^{-2} s\right)$. Evolution of the mode after the first indication of the phase jump could not be extracted with our current analysis from the experimental data. One need dedicated experiments and ECE diagnostic adjusted for the optimal measurements at the mode location for as it was done in previous papers $[8,9]$.

In the present studies, we have focused on analysis of existing typical discharges in the data bases of the DIII-D and ASDEX Upgrade tokamaks. We have analyzed in total 9 discharges from DIII-D and in two cases found long ideal mode. In 3 discharges from ASDEX Upgrade all have a long ideal mode phase. The main plasma parameters of the presented discharges are given in table 1, although they have relatively small influence on the exact details of the seeding process. It is much more important to study the detailed evolution of the plasma rotation profile, the electron temperature profile and the density profile as discussed in the next section.

\begin{tabular}{|l|l|c|c|c|c|c|c|c|}
\hline Tokamak & discharge & $I_{p}[\mathrm{MA}]$ & $q_{95}$ & $B_{t}[\mathrm{~T}]$ & $\beta_{N}$ & $\kappa$ & $\delta_{\text {up }}$ & $\delta_{\text {low }}$ \\
\hline DIII-D & 171114 & 1.6 & 3.29 & 1.9 & 1.8 & 1.825 & 0.07 & 0.65 \\
\hline DIII-D & 169475 & 1.6 & 3.4 & 1.9 & 1.9 & 1.823 & 0.332 & 0.644 \\
\hline AUG & 32940 & 0.8 & 5.2 & 2.5 & 2.17 & 1.757 & 0.118 & 0.493 \\
\hline
\end{tabular}

Table 1. The table shows main parameters of the discharges presented in the paper: plasma current $\left(I_{p}\right)$, safety factor $\left(q_{95}\right)$, magnetic field $\left(B_{t}\right)$, normalized beta $\left(\beta_{N}\right)$, elongation $(\kappa)$, upper $\left(\delta_{u p}\right)$ and lower $\left(\delta_{\text {low }}\right)$ triangularity. 


\section{ANALYSIS OF ROTATION VELOCITIES AND MHD LIMITS}

Before analysis of the rotation profile and MHD limits, we discuss briefly results of MHD simulations which give some hints on the important parameters for the seeding process. Nonlinear simulations of an externally driven $(2,1)$ perturbation predict formation of a dominantly $(2,1)$ kink mode which converts into a $(2,1)$ island on a later time point $[13,14]$. The conversion time depends on the differential plasma rotation between the external perturbation and the electron fluid velocity at the $(2,1)$ resonant surface. When this differential rotation vanishes, the electrons are at rest with respect to the perturbation and magnetic flux efficiently penetrates into the plasma. This flux penetration is accompanied by fast island growth and finally, the non-linear evolution leads to saturation of the island. The seeding depends also on the amplitude of the external perturbation and its duration. These dependences are seen both in experiments and in numerical simulations [15]. The process of the island formation due to an internal event is similar to the seeding of tearing modes by external perturbations in the presence of differential plasma rotation. Non-linear simulations of $(3,2)$ seeding by a sawtooth crash predict formation of $(2,2)$ post-cursor mode directly after the crash [16]. This $(2,2)$ mode drives a dominantly ideal $(3,2)$ kink mode which converts into a $(3,2)$ tearing mode on a longer timescale. The conversion time depends again on the differential plasma rotation between $(2,2)$ perturbation and the electron fluid velocity at the $(3,2)$ resonant surface. In case of zero differential rotation, the island growth is fast from the beginning. Otherwise, there is a phase of slowdown of the plasma rotation at the resonant surface during which the island grows is strongly damped. In these so-called "resonant seeding of tearing mode" cases the seed perturbation has to have the same toroidal mode number. (Poloidal mode numbers are coupled in a torus which enables $(1,1)$ seeding of $(2,1)$ modes or $(2,2)$ seeding of $(3,2)$ modes.)

The other possible variant for a seeding is a modification of the plasma equilibrium by the internal perturbation. In this situation, the MHD equilibrium can have several possible solutions with and without incorporated ideal internal perturbation in the plasma. All these solutions are local energy minima and the energy values are comparable $[17,18,19]$. Thus, the $(3,2)$ kink mode is incorporated into the equilibrium after the crash and converts into the tearing mode on a longer timescale. For this case, the onset frequency of the seeded $(3,2)$ mode is not necessarily dependent on precursor frequencies, like e.g. a $(2,2)$ crash post-cursor, and is determined by plasma profiles around the resonant surface. 
The plasma rotation profiles for DIII-D discharges (\#171114 and \#169475) just before NTM seeding are shown in figure 7.

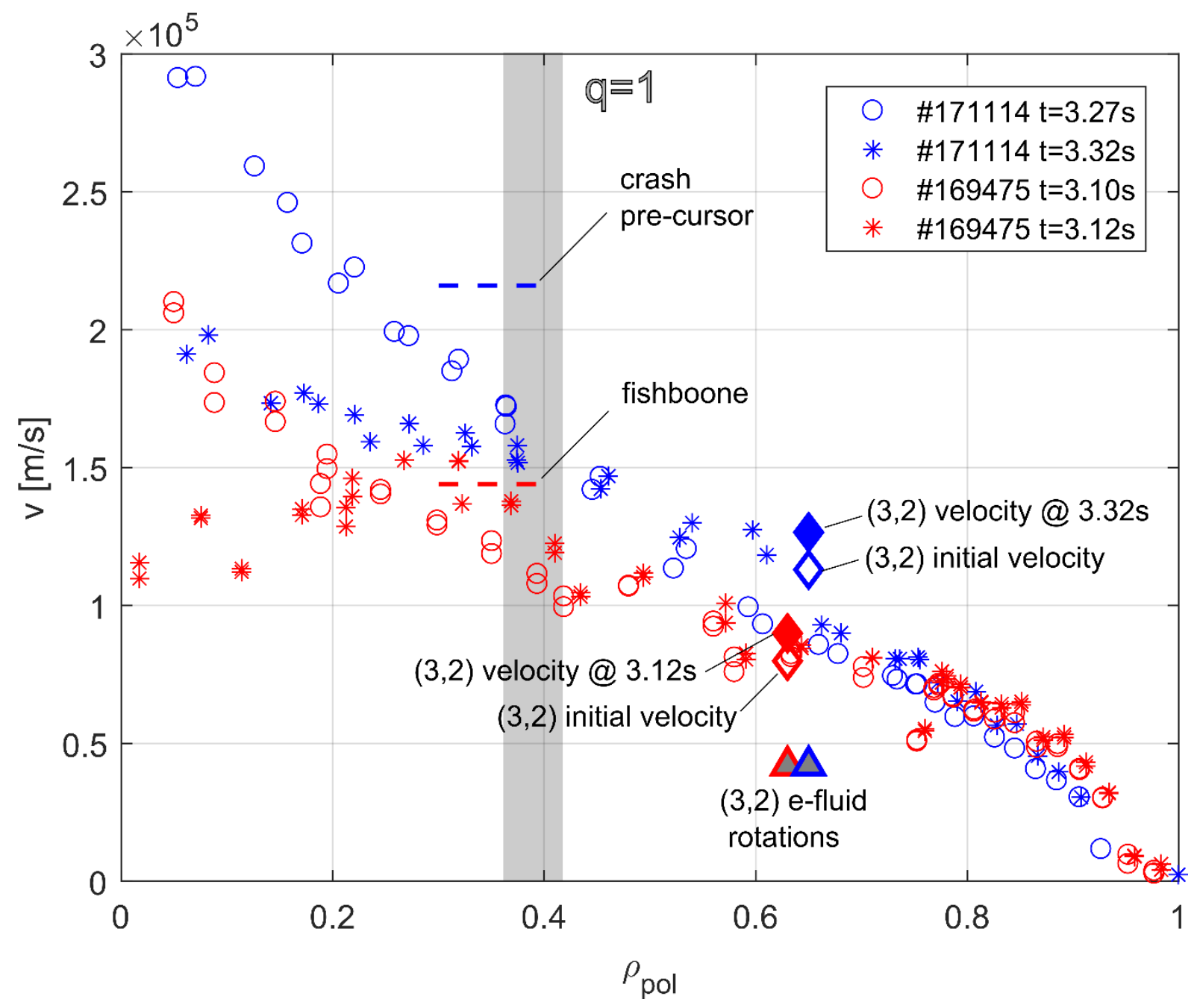

Figure 7. The plasma rotation profiles in DIII-D tokamak before the seeding and slightly after for discharges \#171114 (blue color) and \#169475 (red color) shown in figures 1-4. Position of the $q=1$ surface is indicated by the shaded bar (from ECE inversion). Velocities for seeding events are indicated by dashed lines. $(3,2)$ rotation velocities $\left(v_{(3,2) \text { tor }}\right)$ are shown by diamonds: (i) initial rotation is indicated by empty diamonds; (ii) rotation corresponding to later time are shown with full diamonds. The initial corresponding velocities for electron fluid at the resonant surface, $v_{e f(3,2)}$, are shown with filled triangles.

The observed frequencies of the considered modes in the lab frame $\left(f_{(m, n)}\right)$ are converted to a toroidal velocity assuming toroidal rotation:

$$
v_{(m, n) t o r}=f_{(m, n)} \frac{R_{(m, n)}}{n} 2 \pi
$$


where $R_{(m, n)}$ is the radial position of the mode (m,n) with respect to the main torus axis, $n$ is the toroidal mode number. In the presence of temperature and density gradients, one has to take into account also the electron diamagnetic drift velocity:

$$
v_{\text {dia }, e}=\frac{1}{e n_{e} B^{2}}\left(n_{e} \frac{\partial T_{e}}{\partial r}+T_{e} \frac{\partial n_{e}}{\partial r}\right)
$$

where $T_{e}$ and $n_{e}$ are the temperature and density profiles respectively, $B$ is the total magnetic field and $e$ is the electron charge. In this case, the mode frequency depends on the poloidal and toroidal components of the diamagnetic drift velocity at the resonant surface (Chapter 3 in Ref. [10]):

$$
f_{(m, n)}=\frac{n v_{\text {tor }}}{2 \pi R_{(m, n)}}-\frac{n v_{\text {dia,e,tor }}}{2 \pi R_{(m, n)}}-\frac{m v_{\text {dia,e,pol }}}{2 \pi r_{(m, n)}}
$$

where $v_{d i a, e, t o r}$ and $v_{d i a, e, p o l}$ are poloidal and toroidal component of the $v_{d i a, e}$ at the resonant surface $q=m / n$. In our case, this means that this additional contribution has to be subtracted from the plasma rotation velocity at the resonant surface $\left(v_{p l, t o r}\right)$, taking into account a correction for mode numbers and radial position of the mode $\left(r_{(m, n)}\right)$, to get the relevant rotation of the electron fluid for an $(\mathrm{m}, \mathrm{n})$ mode:

$$
v_{e f(m, n)}=v_{p l, t o r}-v_{d i a, e, t o r}-\frac{m R_{(m, n)}}{n r_{(m, n)}} v_{\text {dia,e,pol }}
$$

This velocity is indicated as electron fluid rotation velocity in figure 7 (filled diamonds) together with $v_{(m, n) t o r}$ (open diamonds). The largest correction here comes from poloidal component of the electron diamagnetic velocity $\left(v_{\text {dia,e,pol }}\right)$.

Comparison of the rotation profiles with the mode frequencies show several important points:

- The precursor MHD velocity (the sawtooth precursor or the fishbone and their harmonics) do not match the onset velocity of the $(3,2)$ mode. Such mismatch between precursor MHD velocity and the mode velocity is a usual observation for $(3,2)$ mode and was already reported from JET[6] and ASDEX Upgrade [5]. Thus, the simple picture of the "resonance seeding" is not confirmed.

- The $(3,2)$ velocities do not match the electron fluid velocities. However, the velocity difference between electron fluid velocity (triangles in figure 7) and the seeded modes (diamonds in figure 7) is larger in the case with long ideal mode (\#171114).

- The case where the initial mode velocity matches the "natural" rotation velocity of the mode after modification of the rotation profile (\#169475) produces an island without long ideal phase (red diamonds in figure 7). In contrary, the case where the initial 
mode rotation velocity is larger than the plasma rotation has the long ideal phase (\#171114). This indicates a possible importance of the plasma rotation for the duration of the ideal phase of $(3,2)$ mode.

These finding show no clear indication for the "resonant seeding" mechanism, but indicate importance of the plasma rotation for the duration of the ideal phase of $(3,2)$ mode. It is clear that further, more detailed, studies are required to clarify the seeding process and the subsequent evolution of $(3,2)$ mode. One has to take into account two types of modification of the rotation profile: (i) during seeding event (sawtooth crash or fishbone); (ii) due to the flattening of the density and temperature inside the island structure. Thus, study of the rotation profile evolution should be done together with corresponding changes in local gradients, which are important for the diamagnetic effect. Present results could not give the final answer for the mechanism of the $(3,2)$ mode seeding.

There is one last important point to check before making any conclusions regarding the mode conversion during seeding. A similar conversion of an ideal mode into a tearing mode is observed in high $\beta_{N}$ plasmas. In these plasmas, the resistive wall mode (RWM) can be unstable above the "no-wall" limit ( chapter 6 in Ref. [10]). This RWM is an ideal kink mode that can change its nature into a tearing mode on a long timescale [20]. Stability calculations with the DCON code [21] show that in our situation the plasma is well below these limits for $n=2$ modes (figure 8). The experimental values of $\beta_{N}$ are even below the "no-wall" stability limits for $\mathrm{n}=1$ modes. Thus, conversion of a RWM could not explain the long-living ideal mode during the seeding process in our case. Presented results show the stability limit for the DIII-D plasmas. Similar stability analysis was made for AUG seeding cases in Ref.[9]. 

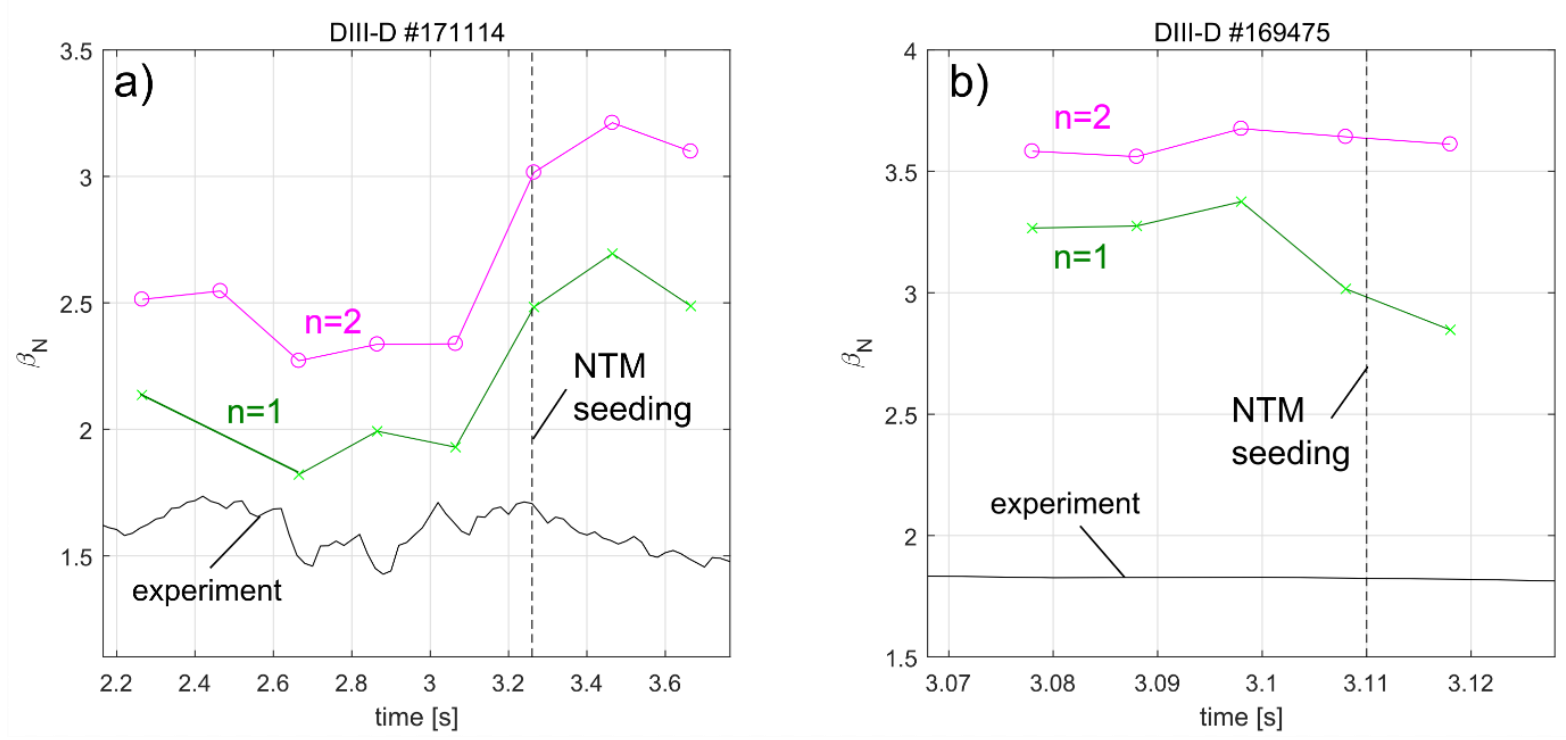

Figure 8. Comparisons of experimental $\beta_{N}$ (black curve) with calculated "no wall" limits for $\mathrm{n}=1$ (green lines) and n=2 (magenta lines) are shown for DIII-D discharges: (a) \#171114, the same as in figures 1 and 2; (b) \#169475, the same as in figures 3 and 4. The vertical dashed line marks the time of the NTM seeding. In both cases, the plasma is well below the "no wall" limit.

\section{CONCLUSIONS}

Experimental observations of $(3,2)$ NTM seeding in DIII-D and ASDEX Upgrade tokamaks shows that in both tokamaks the tearing mode formation can be a long process in the order of milliseconds. The mode amplitude observed in magnetic pickup coils immediately after the trigger event belongs in this case to a dominantly ideal kink mode structure, which converts into a tearing mode with the same helicity and nearly the same externally measured amplitude. Our observations are in good agreement with previous results for $(2,1)$ mode seeding in ASDEX Upgrade tokamak [8,9]. Non-linear MHD simulations for ASDEX Upgrade tokamak also predict conversion from ideal into tearing mode [13-16]. There are also $(3,2)$ NTM seeding cases where no such transition phase can be identified with our method in DIIID tokamak. This is probably connected to the limitation of the necessary analysis (width of the FFT window required for the analysis).

It is important to mention that our analysis does not exclude the existence of a small island incorporated into a larger ideal kink mode immediately after the trigger event. A small island $(1-3 \mathrm{~cm})$ can be invisible for ECE diagnostic in this case because the kink part dominate the signal and one would observe no phase jump in this case. 


\section{ACKNOWLEDGEMENTS}

This work was partially funded by the Max-Planck/Princeton Center for Plasma Physics.

This work has been carried out within the framework of the EUROfusion Consortium and has received funding from the Euratom Research and Training Programme 2014-2018 under grant agreement No. 633053. The views and opinions expressed herein do not necessarily reflect those of the European Commission.

This material is based upon work supported by the U.S. Department of Energy, Office of Science, Office of Fusion Energy Sciences, using the DIII-D National Fusion Facility, a DOE Office of Science user facility, under Awards DE-FC02-04ER54698. DIII-D data shown in this paper can be obtained in digital format by following the links at https://fusion.gat.com/global/D3D_DMP. Disclaimer-This report was prepared as an account of work sponsored by an agency of the United States Government. Neither the United States Government nor any agency thereof, nor any of their employees, makes any warranty, express or implied, or assumes any legal liability or responsibility for the accuracy, completeness, or usefulness of any information, apparatus, product, or process disclosed, or represents that its use would not infringe privately owned rights. Reference herein to any specific commercial product, process, or service by trade name, trademark, manufacturer, or otherwise, does not necessarily constitute or imply its endorsement, recommendation, or favoring by the United States Government or any agency thereof. The views and opinions of authors expressed herein do not necessarily state or reflect those of the United States Government or any agency thereof.

\section{REFERENCES}

[1] T.C. Hender, et al., "Progress in the ITER Physics Basis Chapter 3: MHD stability, operational limits and disruptions", 2007, Nucl. Fusion 47 S128

[2] La Haye R.J. et al, "Dimensionless scaling of the critical beta for onset of a neoclassical tearing mode", 2000 Phys. Plasmas, Vol 7, p.3349

[3] R.J.Buttery, “On the form of NTM onset scalings”,Nucl. Fusion 44 (2004) 678-685

[4] I.T.Chapman, "Empirical scaling of sawtooth period for onset of neoclassical tearing modes", Nucl. Fusion 50 (2010) 102001

[5] A. Gude et.al., „Seed island of neoclassical tearing modes at ASDEX Upgrade“, 1999 Nucl. Fusion 39127

[6] R.J. Buttery et al, "Onset of neoclassical tearing modes on JET”, Nucl. Fusion 43 (2003) 69-83

[7] S Fietz et al," Influence of rotation on the $(m, n)=(3,2)$ neoclassical tearing mode threshold in the ASDEX Upgrade”, Plasma Phys. Control. Fusion 55 (2013) 085010

[8] V. Igochine et al.," Conversion of the dominantly ideal perturbations into a tearing mode after a sawtooth crash" Phys. Plasmas, 21, 110702 (2014)

[9] V. Igochine et al., "Tearing mode formation induced by internal crash events at different $\beta_{\mathrm{N}}$ ", Nucl. Fusion, 57, 036015 (2017)

[10] V. Igochine (ed.) “Active Control of Magneto-hydrodynamic Instabilities in Hot Plasmas”, (Springer, Berlin, 2015)

[11] A. Keller et al., "Analysis methods and conditions for feedback controlled NTM stabilization”,30th EPS Conference., St. Petersburg, 7-11 July 2003 ECA Vol. 27A, P-1.130

[12] M. Reich et al., "Real-time Control of NTMs Using ECCD at ASDEX Upgrade", PPC/P1-26, paper presented at 25th IAEA Int. Conf. on Fusion Energy St Petersburg 2014

[13] D. Meshcheriakov et al., "Tearing mode seeding by external magnetic perturbations", 45nd EPS conf., (2018) 
[14] Q.Yu et.al., "Seed island formation by forced magnetic reconnection”, Nucl. Fusion 52 (2012) 063020 (10pp)

[15] D. Meshcheriakov et al., "Numerical study of tearing mode seeding in tokamak X-point plasma", accepted for publication in Physics of Plasmas

[16] Q.Yu et.al., "NTM Excitation by Sawtooth Crashes and the Suppression of their Onset by Resonant Magnetic Perturbation", Preprint: TH-P5/19, 2018 IAEA Fusion Energy Conference, Gandhinagar

[17] E. Strumberger et al, "Linear stability studies including resistive wall effects with the CASTOR/STARWALL code" 2014 J. Phys.: Conf. Ser. 561012016

[18] E. Strumberger et al.," MHD instabilities in 3D tokamaks” 2014 Nucl. Fusion 54, 064019

[19] W. A. Cooper, J P Graves and O Sauter, "Helical ITER hybrid scenario equilibria”, Plasma Phys. Control. Fusion 53 (2011) 024002

[20] P. Buratti et al.," Kink instabilities in high-beta JET advanced scenarios”, 2012, Nucl. Fusion 52, 023006

[21] A. H. Glasser, "The direct criterion of Newcomb for the ideal MHD stability of an axisymmetric toroidal plasma",Phys. Plasmas, 23 (2016) 072505 